\title{
Fluctuaciones cíclicas en México y en el Estado de México en el contexto del TLCAN: ¿cuáles son los hechos?
}

\author{
Pablo Mejía-Reyes \\ Alberto Mejía-Reyes*
}

\begin{abstract}
This article analyses cyclic fluctuations in the State of Mexico and Mexico from the point of view of the growth cycles in the period between 1993 and 2005. The comparison of our results with those published beforehand indicates changes in the magnitude and nature of the relationships of the cycle as well as in several macroeconomic variables, which suggests the possibility of a structural change during the first half of the nineties. Furthermore, the cycles in Mexico and the State of Mexico are very similar, in particular in their relationship with national variables. The State variables have, at best, a moderate relationship with the indicator of the cycle and they never precede it. This can be evidence of the importance of the national clashes in the State cyclic fluctuations.
\end{abstract}

Keywords: economic cycles, fluctuations, macroeconomic variables, monetary policy.

\section{Resumen}

En este documento se analizan las fluctuaciones cíclicas de México y del Estado de México -para el periodo 1993-2005- empleando el enfoque de los ciclos de crecimiento. La comparación de nuestros resultados con los publicados previamente indica cambios en la magnitud y naturaleza de las relaciones del ciclo y diversas variables macroeconómicas, lo que sugiere la posible existencia de un cambio estructural hacia la primera mitad de los años noventa. Adicionalmente, los ciclos de México y del Estado de México son muy similares, en particular en su relación con las variables nacionales. Por su parte, las variables estatales guardan relaciones moderadas, en el mejor de los casos, con el indicador del ciclo y nunca lo anteceden, lo cual podría evidenciar la importancia de los choques nacionales en las fluctuaciones cíclicas estatales.

Palabras clave: ciclos económicos, fluctuaciones, variables macroeconómicas, política monetaria.

* Universidad Autónoma del Estado de México. Correos-e: pmejr@uaemex.mx, betmej@yahoo.com.mx. 


\section{Introducción*}

El reavivamiento del análisis de los ciclos económicos en la literatura económica internacional, a partir de la crisis de los años setenta, experimentada por la mayoría de los países desarrollados, por una lado, y la alternancia de crisis y recuperaciones económicas en México, también a partir de esa década, han despertado el interés por analizar las fluctuaciones económicas de México hasta fechas relativamente recientes. El análisis de la interrelación del ciclo con otras variables económicas, en la lógica de la popular metodología de Kydland y Prescott (1990), es una de las áreas más importantes (Agénor et al., 2000; Torres, 2002; Mejía, 2003). Un segundo grupo de estudios se ha enfocado a determinar el grado de sincronización internacional del ciclo económico de México con el de Estados Unidos a nivel agregado (Herrera, 2004; Castillo et al., 2004), en tanto que un tercero trata de caracterizar tal sincronización para actividades manufactureras a niveles más o menos desagregadas (Cuevas et al., 2003; Mejía et al., 2006).

Un área que, en nuestra opinión, no ha recibido la atención suficiente es el análisis de la naturaleza de los ciclos estatales o regionales. Este es un tema importante dado que los ciclos subnacionales pueden ser diferentes de los nacionales por factores como la estructura de su producción, las políticas económicas y las condiciones financieras locales, la infraestructura e incluso los factores climáticos, entre otros. ${ }^{1}$ La estructura productiva (y su nivel de competitividad), por ejemplo, determina el grado de integración a las economías nacional e internacional y, en ese sentido, la magnitud de los efectos de las últimas sobre la economía local. Incluso las políticas monetaria y fiscal nacionales, así como los choques tecnológicos, pueden tener un efecto diferente en función de esa misma estructura productiva. Por su parte, la interacción entre las economías local y nacional es directamente proporcional al tamaño de la primera. Por último, la implementación de políticas fiscales locales distintas a las nacionales puede inducir en éstas la presencia de ciclos de distinta naturaleza.

* Los autores agradecen el financiamiento otorgado por la Universidad Autónoma del Estado de México (UAEM) al proyecto con clave 2241/2006U. Asimismo, agradecemos la asistencia de investigación de Jeanett Campos Chávez así como los comentarios de los dictaminadores anónimos.

${ }^{1}$ Para un análisis detallado de los efectos de estos factores, véanse los trabajos de Norrbin y Schlagenhauf (1988), Altonji y Ham (1990), Sala-i-Martin y Sachs (1992), Samolyk (1994) y Clark (1998). 
Entre los pocos trabajos que analizan aspectos de los ciclos económicos de los estados de México se encuentran los de Ponce (2001) y Del Negro y Ponce (1999) quienes, utilizando un modelo de factores y vectores autorregresivos, encuentran que en general los choques nacionales son la fuente principal de las fluctuaciones estatales, aunque en los estados con niveles mayores de ingreso son los choques regionales los de más peso. Por su parte, Cuevas et al. (2003) encuentran que la Región Sur muestra un comportamiento independiente de la evolución económica del resto del país; que la Región Centro es más sensible a las perturbaciones fiscales relacionadas con el precio del petróleo y con otras de índole idiosincrásico; y que la Región Norte se encuentra más vinculada con la evolución de la economía de los Estados Unidos. Por último, en un trabajo reciente, Erquizio (2006) analiza la dinámica de los ciclos de varios estados del país y construye un indicador del ciclo para cada uno de ellos donde efectivamente encuentra diferencias significativas entre los ciclos económicos de los distintos estados.

Con el objeto de contribuir a una mejor comprensión de la dinámica de la economía mexiquense, en este documento se analizan los ciclos económicos del Estado de México para el periodo 1993-2005 y los resultados se comparan con los obtenidos en este mismo ciclo en el ámbito nacional. ${ }^{2}$ En particular, nos interesa establecer qué relación tiene el indicador del ciclo mexiquense con las variables macroeconómicas nacionales más importantes y con las estatales disponibles para determinar si éstas contienen información que permita detectar cambios futuros en la producción real. Un tema central en el análisis es la comparación de estas propiedades con las del ciclo económico nacional.

Para abordar el tema propuesto, este trabajo se divide en dos secciones. En la primera se expone la metodología de los ciclos de crecimiento popularizada por Kydland y Prescott (1990) así como los datos utilizados. En la segunda se presentan los resultados y se discuten las diferencias más importantes entre nuestros resultados y los obtenidos por otros autores para el caso de México y éstos se contrastan con los obtenidos para el Estado de

\footnotetext{
${ }^{2}$ El periodo de análisis se determinó por la disponibilidad de información y puede parecer corto. Sin embargo, es importante tener presente que incluye dos periodos de recesión y dos de expansión (dos ciclos completos), lo que nos da una idea aceptable de la dinámica del ciclo estatal. Más aún, los resultados para el Estado de México se contrastan con los nacionales para tener un referente que nos indique qué tan razonables pueden ser nuestros resultados.
} 
México. Finalmente, se establecen las conclusiones principales y se señalan algunas áreas de investigación futura.

\section{Metodología y variables utilizadas}

El análisis de los ciclos económicos tradicionales se basa en la metodología propuesta por Kydland y Prescott (1990), quienes, siguiendo a Lucas (1977), sostienen que el análisis de las características de los ciclos se puede basar en el estudio de las correlaciones entre las desviaciones de la tendencia del indicador del ciclo (producción) y las correspondientes desviaciones de otras variables. En este documento, la estimación y eliminación de la tendencia se lleva a cabo mediante dos filtros. El primero, propuesto por Hodrick y Prescott (1997), es uno de los más populares, aunque no ha estado exento de críticas. ${ }^{3}$ Aquí adoptamos la versión modificada propuesta por Ravn y Uhling (1997), quienes argumentan que el filtro Hodrick-Prescott ha resistido tanto el debate como el paso del tiempo, por lo que más que proponer un filtro diferente prefieren analizar cómo modificarlo cuando la frecuencia de los datos cambia. ${ }^{4}$

Por otra parte, para evaluar la consistencia de los resultados se aplica un filtro alternativo para eliminar la tendencia, a saber, las tasas anualizadas de crecimiento. Este segundo filtro es más sencillo y tiene dos ventajas: permite obtener series estacionarias (en general) y, a la vez, tiene un significado más cercano a la idea de crecimiento económico. Adicionalmente, ayuda a reducir el ruido de las series mensuales, lo que permite obtener una medida alternativa de series en principio heterogéneas, como pueden ser las de México y las de los Estados Unidos. Así pues, con la aplicación de dos filtros se obtienen medidas alternativas de las fluctuaciones cíclicas de las variables analizadas, las cuales tienen además la peculiaridad de que son estacionarias.

\footnotetext{
${ }^{3}$ Entre las críticas se encuentran las siguientes: puede generar ciclos espurios cuando las series son estacionarias en diferencias, es óptimo sólo en casos especiales y puede generar propiedades de segundo orden extremas en las series resultantes. Véanse Ravn y Uhling (1997) y Ahumada y Garegnani (2000). Por otro lado, como argumenta Canova (1998), todos los filtros tienen limitaciones y las propiedades de los ciclos pueden depender del filtro empleado para eliminar la tendencia.

${ }^{4}$ De manera más específica, ellos proponen modificar el parámetro de suavizamiento de modo que la duración de las fluctuaciones cíclicas se mantenga independientemente de si se usan datos mensuales, trimestrales o anuales. Para datos mensuales, sugieren emplear el parámetro $l=129,600$. En adelante denotaremos este filtro como HPC. También ha sido empleado por Mejía (2003).
} 
Posteriormente se analiza el grado de comovimiento entre el indicador del ciclo $y_{t}$ y el componente cíclico de las demás variables macroeconómicas $x_{i t-j}$ mediante el cálculo de un coeficiente de correlación $p(j)$, para $j=0, \pm 1, \pm 2, \ldots, \pm 9) .{ }^{5}$ A través de este procedimiento se pueden analizar dos aspectos de las relaciones. Primero, es posible determinar la dirección de los comovimientos: cuando los valores contemporáneos de la variable cambian en la misma dirección que los del indicador del ciclo $(p(j)>0)$, se dice que esa variable es procíclica; cuando cambian en dirección contraria $(p(j)<0)$ será contracíclica; y cuando el coeficiente de correlación es cercano a cero, se dice que es no correlacionada. Segundo, se puede detectar la correlación a través del tiempo entre los comovimientos de las variables: si el componente cíclico de una variable se mueve antes que el de la producción, esa variable antecede al ciclo del producto, es decir, $x_{i}$ antecede al ciclo por $j$ periodos si çp(j)ç alcanza su valor máximo para un $j>0$ (rezago); análogamente, si la variable considerada cambia después que el indicador del ciclo, se dice que sigue al ciclo, por lo que çp(j)ç alcanza su valor máximo para una $j<0$ (adelanto). Finalmente, $x_{i t}$ es contemporánea al ciclo del producto cuando çp(j)ç alcanza su valor máximo para $j=0$.

El grado de correlación entre el indicador del ciclo y las demás variables incluidas se considera fuerte si çp(j)ç $>0.6$, moderado si $0.4 \leq c ̧ p(j) c ̧ \leq 0.6$ y débil cuando ç $p(j)$ ç $<0.4$. Este criterio no parece demasiado restrictivo, por lo que pensamos que es aceptable. Más aún, en el análisis enfatizaremos los casos en los que existe una correlación fuerte.

Con respecto a la información estadística, se utilizan datos mensuales desestacionalizados (cuando ha sido necesario) para el periodo 1993.01 a 2005:12. Se emplea el índice de volumen de la producción manufacturera para obtener el indicador del ciclo tanto en el ámbito estatal (Manedo) como en el nacional (Manmx). ${ }^{6}$ En el primer caso, nos vimos obligados a utilizar esta

${ }^{5}$ La correlación se calcula empleando los componentes estacionarios de ambas series obtenidos después de aplicar el mismo filtro. Es importante establecer aquí que los coeficientes no condicionales de correlación no implican relaciones de causalidad en sentido alguno, sino simple asociación entre los movimientos de las variables involucradas.

${ }^{6}$ Convencionalmente, una serie de tiempo se puede descomponer en una tendencia, un componente estacional, un componente estacionario y un componente irregular. El análisis del ciclo económico se concentra en las características del tercer componente, por lo que se emplean datos sin tendencia (ver más adelante) y desestacionalizados. Respecto de las series económicas empleadas, en general se obtuvieron desestacionalizadas de la fuente. En caso contrario, se aplicó el filtro X12-ARIMA contenido en EViews 5.1. Por otra parte, dado que el ciclo es un fenómeno de corto plazo, es 
variable dado que es la única disponible sobre una base mensual, lo cual nos proporciona el suficiente número de datos para llevar a cabo el estudio. Para hacer comparable el análisis, empleamos el mismo indicador en el ámbito nacional. Aunque en principio el periodo de estudio se determinó por la disponibilidad de información para el Estado de México, se puede considerar como relevante dado que a partir de principios de los años noventa se consolidaron las estrategias y características que muy probablemente determinarán la evolución futura de la economía nacional, como la liberalización del comercio y la inversión, el papel del mercado como asignador de recursos y el uso de políticas monetaria y fiscal ortodoxas, entre otras. ${ }^{7}$

Por otro lado, se incluyeron variables correspondientes a los distintos sectores de la economía debido a que uno de los objetivos de un análisis como el presente es documentar los hechos estilizados del ciclo. Sobre esta base, se puede profundizar en la relación entre el ciclo y un conjunto de variables particulares. Más aún, como ocurre frecuentemente en esta literatura, esos hechos empíricos sirvieron como fundamento para el desarrollo de modelos teóricos que pretenden explicarlos. Hasta donde sabemos, un estudio de esta naturaleza no se ha realizado para el ciclo del Estado de México, por lo que determinar esos hechos estilizados es una primera etapa para el análisis sistemático ulterior de relaciones particulares. Además, como punto de comparación, los resultados para este estado se contrastan con los del ciclo nacional: en ambos casos, nuestros resultados se discuten en el contexto de los estudios previos que han documentado la experiencia mexicana, como los de Agénor et al., (2000), Torres (2002) y Mejía (2003).

Así pues, se incorporan variables agregadas correspondientes a lo que hemos llamado sector real, las cuales también se pueden considerar como indicadores alternativos del ciclo, como el índice de formación bruta de capital fijo tres medidas alternativas de

conveniente usar datos de la mayor frecuencia posible. Finalmente, se emplea la producción manufacturera como indicador del ciclo porque, además de ser la única medida de producto disponible, se basa en la Encuesta Industrial Mensual, la cual se levanta en los establecimientos productores haciéndola una medida adecuada para evaluar la producción estatal.

${ }^{7}$ Para un análisis de las implicaciones del Tratado de Libre Comercio para América del Norte (TLCAN) sobre la economía mexicana, se pueden consultar los trabajos de Tornell et al., (2004) y de Kose et al., (2004). Por su parte, algunos estudios argumentan que la economía mexicana se ha sincronizado con la estadounidense solamente a partir de la primera mitad de los años noventa y no antes (Herrera, 2004; Mejía et al., 2006), lo cual sugiere un cambio estructural en esta relación. 
empleo, a saber, la tasa general de desempleo abierto, el número de asegurados y la cantidad de trabajadores afiliados al Instituto Mexicano del Seguro Social (IMSS). Los indicadores fiscales que se analizan son los ingresos y los gastos gubernamentales totales así como la medida del impulso fiscal propuesto por Agénor et al. (2000), que se define como la razón gasto-ingreso del sector público y trata de medir el efecto de la actividad global de gobierno. Entre las variables monetarias y financieras y precios se cuentan los distintos agregados monetarios, como la base monetaria, M1, M2 y M3, las tasas de interés de los Cetes, el costo porcentual promedio, y el índice de precios y cotizaciones de la Bolsa Mexicana de Valores. Entre los indicadores de precios se incluyen el índice nacional de precios al consumidor y el índice de precios al productor, así como la tasa de inflación medida como la tasa anualizada de crecimiento del primero. También se incluyen en este grupo los precios del trabajo, como el salario mínimo y el índice de sueldos y salarios del sector manufacturero. Con respecto al sector externo, se consideran variables nacionales e internacionales. Entre las primeras se cuentan las exportaciones, las importaciones, el saldo de la balanza comercial y el tipo de cambio nominal y real. Por su parte, dado que un elevado volumen del comercio exterior y de la inversión extranjera que ingresa a México proviene de los Estados Unidos, se incluyen dos tipos de variables de ese país que tratan de captar los efectos de su ciclo y de sus decisiones de política. Así, se consideran los efectos del índice de volumen de la producción industrial y manufacturera y de la tasa de interés de los bonos del Tesoro de los Estados Unidos a tres meses, tanto en términos nominales como reales.

Por otro lado, consideramos la relación del ciclo mexiquense con otras variables estatales disponibles, como los índices de compras al mayoreo y al menudeo, los índices de ventas también al mayoreo y al menudeo, producción de electricidad, tasa de desempleo abierto, tasa de desempleo para distintos grupos de edad, personal con diferentes niveles de prestaciones y distintas clasificaciones de población económicamente activa. La nomenclatura de todas las variables previamente mencionadas se encuentra en el Anexo.

En la gráfica i se muestra la evolución de la producción manufacturera del Estado de México y de México. Destacan la desaceleración de principios de los años noventa y la recuperación atribuible a la implementación de políticas expansivas en el año electoral de 1994 (Dornbusch y Werner, 1994), sobre todo en la 
producción estatal. También, llama la atención la rápida recuperación que siguió a la recesión de 1995 y que ha sido ampliamente documentada en la literatura (por ejemplo, Castañeda, 2000). Por último, resalta la recesión de principios de esta década y las dificultades para retomar la senda del crecimiento en los últimos años. También se puede observar la tendencia estimada con el filtro HPC, la cual se emplea para estimar los componentes cíclicos respectivos. ${ }^{8}$

\section{Gráfica I \\ Estado de México: producción manufacturera y tendencia (logaritmos)}

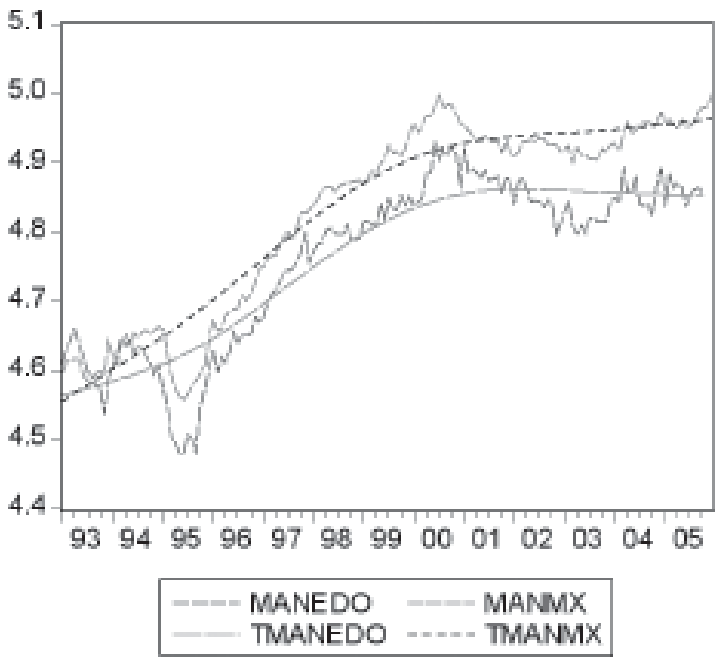

En la gráfica II aparecen los componentes cíclicos y las tasas anualizadas de crecimiento de la producción manufacturera de México y del Estado de México. En general, se observa que las series siguen un comportamiento muy similar, aunque resalta el hecho de que las tasas anualizadas reflejan fluctuaciones más amplias, particularmente durante la rápida recuperación que siguió a la crisis de 1995. Por lo demás, coinciden los episodios de repunte y declinación de la producción: las recesiones de 1995 y

\footnotetext{
${ }^{8}$ Nótese que el filtro HPC estima una tendencia que define el comportamiento de largo plazo y que, según Kydland y Prescott (1990), estaría explicada por los factores de crecimiento. Lo que define el patrón cíclico para este enfoque, entonces, son los movimientos por encima y por debajo de la tendencia. Los componentes cíclicos obtenidos para los casos analizados reflejan claramente los repuntes y declinaciones de la producción.
} 
Gráfica II

Estado de México: componente cíclico y tasa anualizada de crecimiento de la producción

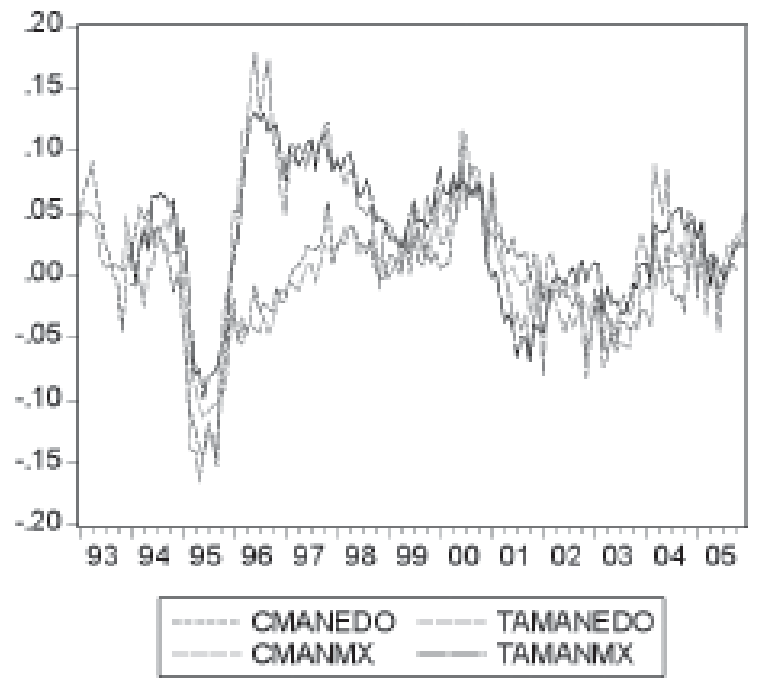

Gráfica III

México y Estado de México: estructura porcentual de la producción manufacturera, 1993-2004 (porcentajes promedio)

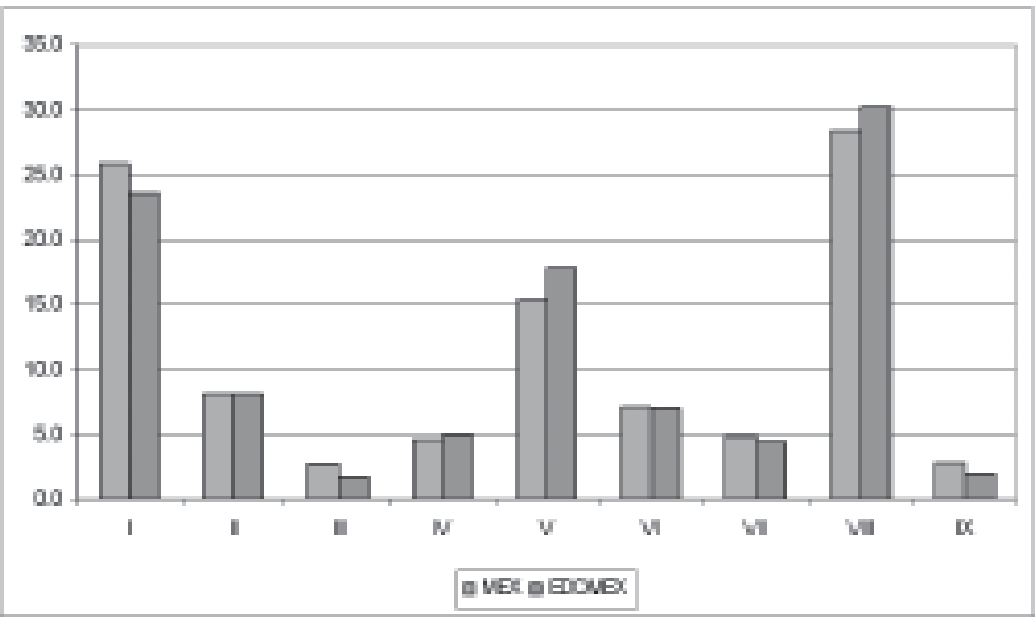


2001-2002, por un lado, y las expansiones de los periodos restantes, por otro.

Un aspecto que llama la atención es la gran similitud entre la producción de ambos espacios geográficos, tanto en los niveles como en los componentes cíclicos y las tasas anualizadas de crecimiento. Como se comentó, la estructura productiva puede determinar la naturaleza de las fluctuaciones cíclicas de una economía local y sus diferencias y semejanzas con respecto a las de la nacional. La gráfica III presenta la estructura de la producción para ambos casos muestra división manufacturera. En primer lugar, respecto de la que la producción manufacturera mexiquense representa un poco más de $16 \%$ de la producción manufacturera nacional, lo cual es bastante significativo si tomamos en cuenta que corresponde a la producción de un solo estado. En segundo lugar, existe una gran similitud en la estructura de ambos espacios territoriales: la producción manufacturera está dominada por tres divisiones que en orden de importancia son las de productos metálicos, maquinaria y equipo; de alimentos, bebidas y tabaco; y de sustancias químicas, derivados del petróleo, productos de caucho y plásticos. Dadas estas características, en general, es de esperarse una relación estrecha entre las fluctuaciones cíclicas de México y las del Estado de México, así como relaciones similares con las otras variables. La verificación de esta conjetura se lleva a cabo en el resto del documento.

\section{Resultados}

En esta sección se dan los resultados obtenidos mediante la aplicación de la metodología ya definida para los casos de México y del Estado de México durante el periodo 1993.01-2005:12. Además, se contrastan nuestros resultados con los aportados por otros autores para el caso de México. ${ }^{9}$ Las relaciones entre las variables macroeconómicas y el ciclo nacional se presentan en el cuadro 1; los resultados correspondientes al Estado de México se observan en el cuadro 2; finalmente, el cuadro 3 contiene la relación entre el ciclo mexiquense y las variables estatales disponibles. Las variables de los cuadros 1 y 2 se organizaron en diferentes grupos; el orden en que se presentan es como sigue: variables de lo que hemos llamado sector real, variables fiscales, variables monetarias y financieras y precios y variables del sector externo.

\footnotetext{
${ }^{9}$ Se consideran los trabajos de Agénor et al. (2000), Torres (2002), Alper (2002) y Mejía (2003). Para no hacer repetitiva la exposición, se enfatizan sólo los casos en los que los resultados difieren sustancialmente.
} 


\section{Cuadro 1 \\ México: correlación de las fluctuaciones de la producción manufacturera con la de otras variables macroeconómicas nacionales}

\begin{tabular}{|c|c|c|c|c|c|c|c|c|}
\hline \multirow[b]{2}{*}{ Variable } & \multicolumn{4}{|c|}{ Hodrick-Prescott corregido } & \multicolumn{4}{|c|}{ Tasa anualizada de crecimiento } \\
\hline & Dirección & Coeficiente & $\begin{array}{c}\text { e Adelanto/ } \\
\text { rezago }\end{array}$ & Ciclic & Dirección & Coeficiente & $\begin{array}{l}\text { Adelanto/ } \\
\text { rezago }\end{array}$ & Ciclic \\
\hline Indmx & Contem & 0.9816 & 0 & Pro & Contem & 0.9763 & 0 & Pro \\
\hline Adel & Antecede & 0.7898 & 1 & Pro & Antecede & 0.7633 & 1 & Pro \\
\hline Invfb & Contem & 0.8748 & 0 & Pro & Contem & 0.8540 & 0 & Pro \\
\hline Des & Sigue & -0.7928 & 1 & Contra & Sigue & -0.8044 & 1 & Contra \\
\hline Ase & Sigue & 0.8660 & 2 & Pro & Sigue & 0.8341 & 3 & Pro \\
\hline Tas & Sigue & 0.9052 & 3 & Pro & Antecede & -0.5849 & 9 & Contra \\
\hline GP & Antecede & 0.3324 & 6 & Pro & Antecede & 0.4467 & 3 & Pro \\
\hline IP & Antecede & 0.3407 & 3 & Pro & Antecede & 0.4859 & 4 & Pro \\
\hline IF & Antecede & -0.1373 & 9 & Pro & Antecede & -0.1724 & 9 & Pro \\
\hline BMN & Contem & 0.8077 & 0 & Pro & Contem & 0.8354 & 0 & Pro \\
\hline BMR & Sigue & 0.5500 & 3 & Pro & Antecede & -0.5238 & 9 & Pro \\
\hline $\mathrm{M} 1 \mathrm{~N}$ & Antecede & 0.7452 & 2 & Pro & Antecede & 0.8150 & 1 & Pro \\
\hline $\mathrm{M} 1 \mathrm{R}$ & Sigue & 0.6245 & 1 & Pro & Contem & 0.5200 & 0 & Pro \\
\hline$M 2 N$ & Antecede & 0.7751 & 8 & Pro & Antecede & 0.7264 & 7 & Pro \\
\hline $\mathrm{M} 2 \mathrm{R}$ & Sigue & 0.5709 & 2 & Pro & Sigue & 0.4944 & 1 & Pro \\
\hline$M 3 N$ & Contem & 0.7698 & 0 & Pro & Antecede & 0.6818 & 1 & Pro \\
\hline $\mathrm{M} 3 \mathrm{R}$ & Sigue & 0.5713 & 3 & Pro & Antecede & -0.4715 & 9 & Pro \\
\hline Ce28n & Antecede & -0.5361 & 3 & Contra & Antecede & -0.5970 & 2 & Contra \\
\hline $\mathrm{Ce} 28 \mathrm{r}$ & Antecede & 0.3593 & 9 & Contra & Contem & -0.4480 & 0 & Contra \\
\hline CPPN & Antecede & -0.2975 & 2 & Contra & Antecede & -0.4056 & 2 & Contra \\
\hline CPPR & Antecede & -0.3400 & 3 & Contra & Antecede & -0.4881 & 3 & Contra \\
\hline BMV & Antecede & 0.7093 & 3 & Pro & Antecede & 0.6856 & 3 & Pro \\
\hline INPC & Antecede & 0.7014 & 8 & Pro & Antecede & 0.7438 & 9 & Pro \\
\hline IPP & Antecede & 0.5773 & 9 & Contra & Antecede & 0.6680 & 9 & Pro \\
\hline INF & Antecede & -0.5409 & 3 & Contra & Antecede & -0.5926 & 2 & Contra \\
\hline Smin & Antecede & 0.6283 & 8 & Pro & Antecede & 0.6636 & 9 & Pro \\
\hline Sminr & Sigue & 0.4647 & 4 & Pro & Antecede & -0.4936 & 9 & Pro \\
\hline Immn & Antecede & 0.4314 & 6 & Pro & Antecede & 0.5733 & 9 & Pro \\
\hline Immr & Antecede & -0.5090 & 9 & Pro & Antecede & -0.6111 & 9 & Pro \\
\hline $\mathrm{x}$ & Sigue & 0.3281 & 1 & Pro & Contem & 0.3288 & 0 & Pro \\
\hline M & Sigue & 0.8959 & 1 & Pro & Contem & 0.8998 & 0 & Pro \\
\hline $\mathrm{BC}$ & Sigue & -0.6550 & 1 & Contra & Sigue & -0.6094 & 1 & Contra \\
\hline $\mathrm{TCN}$ & Contem & -0.4816 & 0 & Contra & Contem & -0.3486 & 0 & Contra \\
\hline ITCR & Antecede & -0.6084 & 2 & Contra & Antecede & -0.6114 & 2 & Contra \\
\hline TI & Antecede & -0.6694 & 1 & Contra & Antecede & -0.6089 & 1 & Contra \\
\hline Maneu & Sigue & 0.7595 & 1 & Pro & Sigue & 0.6824 & 1 & Pro \\
\hline Indeu & Sigue & 0.7594 & 1 & Pro & Sigue & 0.6793 & 1 & Pro \\
\hline TВT3N & Sigue & 0.5358 & 4 & Pro & Sigue & 0.4598 & 4 & Pro \\
\hline TBT3R & Sigue & 0.4615 & 4 & Pro & Sigue & 0.3812 & 4 & Pro \\
\hline
\end{tabular}

Ciclic se refiere a ciclicalidad, contem a contemporáneo, pro a procíclico y contra a contracíclico.

La comparación de los resultados de los primeros dos cuadros sugiere una similitud significativa entre el ciclo mexicano y el mexiquense. En primer lugar, llama la atención la consistencia de los resultados a través del filtro empleado para eliminar la tendencia en la gran mayoría de los casos. ${ }^{10}$ Asimismo, existe

${ }^{10}$ Las únicas excepciones son la base monetaria real, M3 real, el salario mínimo real, el impulso fiscal y el número de trabajadores asegurados en el IMSs (Tas) en el caso del Estado de México, en tanto que para México son las mismas variables excepto porque la tasa de interés real de los Cetes sustituye al impulso fiscal. 
Cuadro 2

\section{Estado de México: correlación de las fluctuaciones de la producción manufacturera con la de otras variables macroeconómicas nacionales}

\begin{tabular}{|c|c|c|c|c|c|c|c|c|}
\hline \multirow[b]{2}{*}{ Variable } & \multicolumn{4}{|c|}{ Hodrick-Prescott corregido } & \multicolumn{4}{|c|}{ Tasa anualizada de crecimiento } \\
\hline & Dirección & Coeficiente & $\begin{array}{c}\text { Adelanto/ } \\
\text { rezago }\end{array}$ & Ciclic & Dirección & Coeficiente & $\begin{array}{c}\text { Adelanto/ } \\
\text { rezago }\end{array}$ & Ciclic \\
\hline Manmx & Contem & 0.8564 & 0 & Pro & Contem & 0.8859 & 0 & Pro \\
\hline Indmx & Contem & 0.8647 & 0 & Pro & Sigue & 0.8941 & 1 & Pro \\
\hline Adel & Contem & 0.7771 & 0 & Pro & Contem & 0.7587 & 0 & Pro \\
\hline Invfb & Sigue & 0.8601 & 1 & Pro & Sigue & 0.8627 & 1 & Pro \\
\hline Des & Sigue & -0.8103 & 2 & Contra & Sigue & -0.8063 & 2 & Contra \\
\hline Ase & Sigue & 0.7862 & 3 & Pro & Sigue & 0.7765 & 4 & Pro \\
\hline Tas & Sigue & 0.7745 & 2 & Pro & Antecede & -0.3677 & 9 & Pro \\
\hline GP & Antecede & 0.3885 & 7 & Pro & Antecede & 0.4816 & 6 & Pro \\
\hline IP & Antecede & 0.3689 & 5 & Pro & Antecede & 0.4360 & 4 & Pro \\
\hline IF & Antecede & -0.1743 & 9 & Contra & Antecede & -0.2331 & 9 & Pro \\
\hline BMN & Sigue & 0.7008 & 1 & Pro & Contem & 0.7223 & 0 & Pro \\
\hline BMR & Sigue & 0.5133 & 5 & Pro & Antecede & -0.5156 & 9 & Pro \\
\hline $\mathrm{M} 1 \mathrm{~N}$ & Contem & 0.7938 & 0 & Pro & Contem & 0.8257 & 0 & Pro \\
\hline $\mathrm{M} 1 \mathrm{R}$ & Sigue & 0.6726 & 2 & Pro & Sigue & 0.5861 & 2 & Pro \\
\hline$M 2 N$ & Antecede & 0.7349 & 7 & Pro & Antecede & 0.6345 & 7 & Pro \\
\hline $\mathrm{M} 2 \mathrm{R}$ & Sigue & 0.5725 & 3 & Pro & Sigue & 0.5773 & 3 & Pro \\
\hline $\mathrm{M} 3 \mathrm{~N}$ & Contem & 0.7342 & 0 & Pro & Contem & 0.6059 & 0 & Pro \\
\hline $\mathrm{M} 3 \mathrm{R}$ & Sigue & 0.5943 & 5 & Pro & Antecede & -0.5267 & 9 & Pro \\
\hline $\mathrm{Ce} 28 \mathrm{n}$ & Antecede & -0.5997 & 1 & Contra & Antecede & -0.6355 & 1 & Contra \\
\hline $\mathrm{Ce} 28 \mathrm{r}$ & Antecede & 0.4983 & 9 & Contra & Antecede & 0.5377 & 9 & Contra \\
\hline CPPN & Antecede & -0.3950 & 1 & Contra & Antecede & -0.4606 & 1 & Contra \\
\hline CPPR & Antecede & -0.4253 & 2 & Contra & Antecede & -0.5207 & 1 & Contra \\
\hline BMV & Antecede & 0.6736 & 2 & Pro & Antecede & 0.6307 & 2 & Pro \\
\hline INPC & Antecede & 0.7165 & 7 & Pro & Antecede & 0.7489 & 9 & Pro \\
\hline IPP & Antecede & 0.7003 & 9 & Contra & Antecede & 0.7060 & 9 & Contra \\
\hline INF & Antecede & -0.6193 & 2 & Contra & Antecede & -0.6518 & 1 & Contra \\
\hline Smin & Antecede & 0.6574 & 7 & Pro & Antecede & 0.6220 & 9 & Pro \\
\hline Sminr & Sigue & 0.5584 & 6 & Pro & Antecede & -0.5001 & 9 & Pro \\
\hline Immn & Antecede & 0.5272 & 5 & Pro & Antecede & 0.5498 & 9 & Pro \\
\hline Immr & Antecede & -0.5765 & 9 & Pro & Antecede & -0.5920 & 9 & Pro \\
\hline $\mathrm{x}$ & Antecede & 0.3212 & 9 & Pro & Antecede & 0.3444 & 9 & Pro \\
\hline M & Sigue & 0.8236 & 1 & Pro & Sigue & 0.8417 & 1 & Pro \\
\hline $\mathrm{BC}$ & Sigue & -0.6826 & 1 & Contra & Sigue & -0.6878 & 2 & Contra \\
\hline TCN & Sigue & -0.5516 & 3 & Contra & Sigue & -0.4471 & 2 & Contra \\
\hline ITCR & Antecede & -0.6517 & 2 & Contra & Antecede & -0.6655 & 1 & Contra \\
\hline TI & Sigue & -0.7167 & 1 & Contra & Sigue & -0.6938 & 1 & Contra \\
\hline Maneu & Sigue & 0.5190 & 1 & Pro & Sigue & 0.4500 & 3 & Pro \\
\hline Indeu & Sigue & 0.5195 & 1 & Pro & Sigue & 0.4456 & 1 & Pro \\
\hline TBT3N & Sigue & 0.2758 & 5 & Pro & Sigue & 0.2246 & 5 & Pro \\
\hline TВT3R & Sigue & 0.3015 & 6 & Pro & Sigue & 0.2296 & 6 & Pro \\
\hline
\end{tabular}

Ciclic se refiere a ciclicalidad, contem a contemporáneo, pro a procíclico y contra a contracíclico.

una gran semejanza en el conjunto de variables con las que los indicadores del ciclo tienen una correlación fuerte (las cuales aparecen en negritas en los cuadros 1 y 2).

Nótese que todas las variables del sector real presentan una relación fuerte con los indicadores del ciclo nacional y estatal. La fuerte relación con la producción industrial se explica por la alta proporción que representa la manufactura. Por su parte, como 
se ha documentado ampliamente, la inversión también está estrechamente vinculada al ciclo: es procíclica, contemporánea y su máximo coeficiente de correlación es el más alto en la muestra (excluyendo el de la producción industrial). ${ }^{11}$ A su vez, la relación con el índice adelantado del Instituto Nacional de Estadística Geografía e Informática (INEGI) también es la esperada, excepto porque la magnitud del periodo con el que este indicador antecede al del ciclo nacional se reduce si lo comparamos con lo informado previamente en la literatura. ${ }^{12}$ Obsérvese también que este índice adelantado es contemporáneo al indicador del ciclo mexiquense, lo cual sugiere que no contiene información para predecir al último. Las relaciones con los indicadores del empleo también son convencionales: la tasa de desempleo es contracíclica y el número de asegurados en el IMss es procíclico. Además, los resultados muestran que el empleo sigue a la producción, esto sugiere que la causalidad puede ir de la última hacia el primero. ${ }^{13}$

Entre las variables monetarias y financieras y precios destacan los agregados monetarios nominales, que tienen una relación fuerte con los indicadores del ciclo nacional y local. En general, anteceden o son contemporáneos al ciclo, la correlación de máximo valor absoluto es positiva y la correlación entre los valores contemporáneos indica que estas variables son procíclicas. Estos resultados sugieren la posibilidad de que el dinero sea exógeno con respecto al ciclo nacional, como se deriva de los modelos convencionales de economía abierta con tipo de cambio flexible y son consistentes con el régimen de política monetaria implementada en México para la mayor parte del periodo de estudio. ${ }^{14}$

Por su parte, la inflación antecede al indicador del ciclo por un lapso máximo de tres periodos y es contracíclica, pero sólo tiene una relación negativa fuerte en el caso del Estado de México. Es decir, al parecer los efectos nocivos de la inflación sobre

\footnotetext{
${ }^{11}$ Como es bien sabido, un aspecto que surge siempre que se analiza la relación entre la inversión y la producción es el de la endogeneidad de la primera, es decir, ambas variables se retroalimentan y pueden, de hecho, depender del mismo conjunto de variables.

${ }^{12}$ Mejía (2003) y Phillips et al. (1996) informan periodos de dos y cuatro meses, respectivamente.

${ }^{13}$ Es interesante observar que el número de trabajadores afiliados al IMss (Tas) no tiene una relación consistente con el indicador del ciclo del país, ni con el del estado, lo que resulta por demás extraño.

${ }^{14}$ También difieren de los informados en estudios previos por Agénor et al. (2000), Torres (2002) y Mejía (2003), quienes encuentran que los indicadores monetarios en términos reales son los que están vinculados al ciclo. Además, dado que los agregados monetarios son contemporáneos o siguen al indicador del ciclo, sugieren la posibilidad de que el dinero sea endógeno.
} 
la actividad productiva nacional, documentados en otros estudios, disminuyeron junto con el nivel de la tasa de inflación, aunque se mantiene la relación negativa entre ambas variables. Por su parte, y más de acuerdo con lo obtenido en países desarrollados (Chadha y Prasad, 1994; Stock y Watson, 1998), el nivel de precios antecede y está positivamente relacionado con los indicadores del ciclo de ambos espacios territoriales. La relación hallada aquí entre nivel de precios y el indicador del ciclo difiere sustancialmente de lo informado en otros estudios y puede indicar un cambio de fondo en la economía mexicana. Los efectos adversos de la inflación y del nivel de precios sobre la producción es probable que estén presentes cuando la primera es elevada, tal como ocurrió en la década de los ochenta, periodo incluido en la mayoría de los estudios sobre los ciclos de México. Sin embargo, una vez que la inflación baja a valores de un dígito, es posible que sus efectos adversos se diluyan y los precios y la producción puedan vincularse más en la lógica de los modelos convencionales de oferta y demanda agregadas. Nótese que la similitud de estos resultados con los obtenidos para el salario mínimo nominal -que es la única medida de ingresos de los trabajadores con la que el ciclo tiene una relación fuerte-, podría indicar que la evolución de éste sigue al nivel de precios dada la política de aumentos salariales en función de la inflación esperada. ${ }^{15}$

Finalmente, el índice de precios y cotizaciones de la Bolsa Mexicana de Valores (BMV) antecede y es procíclico con respecto a los dos indicadores del ciclo, lo cual sugiere que puede contener información para preveer el comportamiento de la producción, como argumenta Ron (2001). ${ }^{16}$

Las variables del comercio exterior muestran una fuerte relación solamente en los casos de las importaciones y de la balanza comercial, aunque es muy probable que la dinámica de la última esté condicionada por la de las primeras, sobre todo si se toma en cuenta la baja correlación entre el indicador del ciclo y las exportaciones. Así pues, como sugieren los modelos de crecimiento con restricciones de balanza de pagos (Casar et al., 1985; Loría y Fuji, 1997), el crecimiento genera una reducción en las exportaciones netas debido a la dependencia del aparato pro-

15 También en este caso los resultados difieren sustancialmente de los presentados en los trabajos citados.

${ }^{16}$ Nótese que una relación como ésta difiere con respecto al perfil temporal y el signo de las presentadas previamente. La razón podría estar asociada con un papel más activo y significativo de la BMV en la determinación de la inversión y la producción en los años recientes. 
ductivo de la importación de insumos, maquinaria y equipo y la incapacidad de las exportaciones para financiarlas. Por su parte, la relación entre el indicador del ciclo con el tipo de cambio se explica con base en los modelos neoestructuralistas que derivan efectos adversos de las variaciones en el tipo de cambio real sobre la actividad productiva, debido a sus efectos nocivos sobre el costo de las importaciones y el servicio de la deuda en términos de moneda doméstica, entre otros (Cottani et al., 1990; Kamin y Rogers, 2000). ${ }^{17}$ Por último, se encuentra una relación negativa con los términos de intercambio, en contra de lo que sugiere la teoría. Una posible explicación de estos resultados es la poca contribución al crecimiento económico del incremento sostenido que los términos de intercambio experimentaron a partir de la segunda parte de los años noventa, derivado del aumento en los precios internacionales del petróleo.

Por su parte, con respecto a las variables del sector externo relacionadas con la economía estadounidense, se puede decir que solamente existe una asociación fuerte entre el indicador del ciclo de México y el del vecino del norte, tanto si se mide con la producción manufacturera como si se hace con la industrial. De hecho, el ciclo de Estados Unidos antecede al de México por un lapso tan corto (un periodo) que se puede aceptar la argumentación de que los ciclos de México y de los Estados Unidos se han sincronizado en fechas recientes (Torres y Vela, 2003; Herrera, 2004; Castillo et al., 2004). Los valores correspondientes para el Estado de México sugieren una relación de la misma naturaleza, pero de un menor grado, ya que los coeficientes obtenidos indican únicamente una correlación moderada. El resto de las variables estadounidenses tienen una relación moderada, en el mejor de los casos, con el ciclo mexicano y el mexiquense, lo cual es consistente con lo informado en la literatura.

El resto de las variables analizadas guardan una relación moderada, en el mejor de los casos, con los indicadores del ciclo. En primer lugar, destacan los indicadores fiscales que son procíclicos y tienen coeficientes de correlación relativamente bajos. Hay, sin embargo, un aspecto que vale la pena destacar: a diferencia de lo encontrado por Mejía (2003), el ingreso y el gasto públicos anteceden a la producción, lo cual imprime un carácter exógeno

\footnotetext{
${ }^{17}$ Este resultado es interesante porque muestra que la relación negativa entre ambas variables no se explica exclusivamente por la coincidencia en el pasado de episodios de recesión acompañados de grandes devaluaciones, sino más bien sugiere un rasgo que está presente también en periodos de relativa estabilidad económica.
} 
a la política fiscal, aunque el hecho de que estos indicadores sean procíclicos implica un papel limitado en términos de política de estabilización. Finalmente, nótese que los posibles efectos del impulso fiscal sobre la producción son más bien limitados. ${ }^{18}$ Por su parte, las tasas de interés, tanto nominales como reales, parecen no ser relevantes en la explicación de las fluctuaciones cíclicas, como se ha argumentado en la literatura. El modesto papel de este canal de la política monetaria podría explicarse por la ausencia de crédito al sector privado, por lo que las decisiones de producción e inversión se toman al margen de las condiciones crediticias (entre las que destacan obviamente el costo del crédito a las actividades productivas). ${ }^{19}$

Por otro lado, se ha analizado la relación entre el ciclo mexiquense y una serie de variables estatales disponibles entre las que se encuentran índices de compras y ventas al mayoreo y menudeo, producción de electricidad, tasas de desempleo por sexo y grupo de edad y diferentes grupos de población económicamente activa. Como se ve en el cuadro 3, los máximos valores de los coeficientes de correlación sugieren una asociación moderada, en el mejor de los casos, para un pequeño grupo de variables. Las ventas al mayoreo y la producción de electricidad son procíclicas, en tanto que las tasas de desempleo son contracíclicas. Particularmente, las tasas de desempleo estatal y la de hombres presentan los coeficientes más elevados, así como las tasas de los trabajadores que tienen entre 25 y 44 años. Entre las razones que podrían explicar por qué solamente estos grupos parecen estar asociados al ciclo, se encuentran las dificultades de la gente joven para ingresar al mercado laboral (Navarrete, 2001) y los problemas de las personas de edad madura para reinsertarse en este mercado una vez que son despedidos de sus empleos. Sin lugar a dudas esta relación debe investigarse con mayor profundidad.

También es importante destacar que ninguna de estas variables antecede al indicador del ciclo mexiquense, por lo que ninguna de ellas parece contener información para pronosticar la evolución futura de la producción. El perfil temporal de las relaciones con las tasas de desempleo es similar al del caso nacional, por lo que aquí también se podría concluir que muy probable-

${ }^{18}$ Braun (2001) presenta resultados similares con respecto al gasto y a los ingresos públicos para un conjunto de países, en tanto que Gavin y Perotti (1997) encuentran una relación similar entre producción y saldo fiscal para un grupo de países latinoamericanos.

${ }^{19}$ Para una exposición más amplia, así como las referencias ahí citadas, véase Mejía (2003). 


\section{Cuadro 3}

\section{Estado de México: correlación de las fluctuaciones de la producción manufacturera con la de otras variables macroeconómicas estatales}

\begin{tabular}{|c|c|c|c|c|c|c|c|c|}
\hline \multirow[b]{2}{*}{ Variable } & \multicolumn{3}{|c|}{ Hodrick-Prescott corregido } & \multirow[b]{2}{*}{ Ciclic } & \multicolumn{4}{|c|}{ Tasa anualizada de crecimiento } \\
\hline & Dirección & Coeficiente & $\begin{array}{l}\text { Sigueto/ } \\
\text { antecede }\end{array}$ & & Dirección & Coeficiente & $\begin{array}{l}\text { Sigueto/ } \\
\text { antecede }\end{array}$ & Ciclic \\
\hline Indcma & Sigue & 0.5401 & 1 & Pro & Contem & 0.3808 & 0 & Pro \\
\hline Indcme & Sigue & 0.5561 & 2 & Pro & Sigue & 0.2576 & 5 & Pro \\
\hline Indvma & Contem & 0.6095 & 0 & Pro & Contem & 0.4755 & 0 & Pro \\
\hline Indvme & Sigue & 0.5115 & 4 & Pro & Antecede & -0.3809 & 9 & Pro \\
\hline Elec & Sigue & 0.4572 & 2 & Pro & Sigue & 0.4484 & 3 & Pro \\
\hline Asalsinpres & Sigue & 0.1825 & 2 & Pro & Antecede & -0.2491 & 9 & Pro \\
\hline De12mas & Sigue & -0.3182 & 7 & Contra & Sigue & -0.2853 & 7 & Contra \\
\hline Desedo & Sigue & -0.5223 & 3 & Contra & Sigue & -0.5631 & 3 & Contra \\
\hline De12a19 & Sigue & -0.3625 & 2 & Contra & Sigue & -0.3259 & 2 & Contra \\
\hline De20a24 & Sigue & -0.2691 & 2 & Contra & Contem & -0.2499 & 0 & Contra \\
\hline De25a34 & Contem & -0.4231 & 0 & Contra & Sigue & -0.4314 & 2 & Contra \\
\hline De35a44 & Sigue & -0.4465 & 3 & Contra & Sigue & -0.4646 & 2 & Contra \\
\hline De45mas & Sigue & -0.3290 & 7 & Contra & Sigue & -0.2969 & 6 & Contra \\
\hline Desh & Sigue & -0.4891 & 3 & Contra & Sigue & -0.5234 & 3 & Contra \\
\hline Desm & Antecede & -0.3404 & 3 & Contra & Sigue & -0.3651 & 3 & Contra \\
\hline Ocsinprest & Antecede & 0.1650 & 1 & Pro & Antecede & 0.2618 & 1 & Pro \\
\hline PEA & Sigue & -0.2158 & 6 & Contra & Sigue & -0.3638 & 5 & Contra \\
\hline PEAH & Antecede & -0.1262 & 3 & Contra & Contem & -0.3556 & 0 & Contra \\
\hline PEAM & Sigue & -0.2859 & 9 & Contra & Sigue & -0.4555 & 5 & Contra \\
\hline PNEA & Sigue & 0.2158 & 6 & Pro & Sigue & 0.3638 & 5 & Pro \\
\hline Pneacex & Sigue & -0.1867 & 5 & Contra & Sigue & -0.2594 & 5 & Contra \\
\hline Pneasex & Sigue & 0.1867 & 5 & Pro & Sigue & 0.2594 & 5 & Pro \\
\hline Pneacasa & Antecede & -0.1365 & 5 & Contra & Sigue & -0.1296 & 1 & Contra \\
\hline Pneadisp & Sigue & 0.3960 & 6 & Pro & Antecede & -0.3863 & 8 & Contra \\
\hline Pneanodis & Sigue & -0.3960 & 6 & Contra & Antecede & 0.3863 & 8 & Pro \\
\hline Pneapens & Sigue & -0.1795 & 8 & Contra & Sigue & -0.1450 & 9 & Contra \\
\hline Pneastu & Sigue & -0.1023 & 2 & Contra & Sigue & -0.1444 & 5 & Contra \\
\hline Trest1a5 & Antecede & -0.2252 & 8 & Pro & Antecede & -0.2987 & 9 & Pro \\
\hline Trmensal48 & Antecede & -0.3167 & 5 & Contra & Antecede & -0.5163 & 1 & Contra \\
\hline
\end{tabular}

Ciclic se refiere a ciclicalidad, contem a contemporáneo, pro a procíclico y contra a contracíclico.

mente el empleo es una variable endógena. Por último, obsérvese que el resto de las variables incluidas en el cuadro 3 guardan una relación débil con el indicador del ciclo.

\section{Conclusiones}

En este documento se analizó la dinámica cíclica de la producción manufacturera del Estado de México y su relación con otras variables macroeconómicas nacionales y estatales siguiendo la metodología de los ciclos de crecimiento propuesta por Kydland y Prescott (1990). Se dio especial peso a la comparación de estas relaciones con las que se encuentran para el ciclo nacional, con el objeto de determinar hasta qué punto ambos ciclos son similares y de determinar las variables que pueden contener informa- 
Anexo

Variables y notación

\begin{tabular}{|c|c|}
\hline \multicolumn{2}{|l|}{ NACIONALES } \\
\hline Manufacturas & Manmx \\
\hline Volumen de la industria & Indmx \\
\hline Índice adelantado & Adel \\
\hline Índice de volumen de la inversión fija bruta & Invfb \\
\hline Tasa de desempleo abierto & Des \\
\hline Total de asegurados al IMSS & Ase \\
\hline Trabajadores asegurados al IMSS & Tas \\
\hline Gasto público & GP \\
\hline Ingreso público & IP \\
\hline Impulso fiscal & IF \\
\hline Base monetaria nominal & BMN \\
\hline Base monetaria real & BMR \\
\hline M1 nominal & $\mathrm{M} 1 \mathrm{~N}$ \\
\hline M1 real & M1R \\
\hline M2 nominal & $\mathrm{M} 2 \mathrm{~N}$ \\
\hline M2 real & $\mathrm{M} 2 \mathrm{R}$ \\
\hline M3 nominal & $\mathrm{M} 3 \mathrm{~N}$ \\
\hline M3 real & $\mathrm{M} 3 \mathrm{R}$ \\
\hline Cetes 28 días nominal & Ce28n \\
\hline Cetes 28 días real & Ce28r \\
\hline Costo porcentual promedio nominal & CPPN \\
\hline Costo porcentual promedio real & CPPR \\
\hline Índice de precios de la Bolsa Mexicana de Valores & BMV \\
\hline Índice nacional de precios consumidor & INPC \\
\hline Índice de precios al productor & IPP \\
\hline Inflación & Inf \\
\hline Salario mínimo nominal & Smin \\
\hline Salario mínimo real & Sminr \\
\hline Índice de renumeraciones de industria manufacturera nominal & Immn \\
\hline Índice de renumeraciones de industria manufacturera real & Immr \\
\hline Exportaciones & $\mathrm{x}$ \\
\hline Importaciones & м \\
\hline Balanza comercial & $\mathrm{BC}$ \\
\hline Tipo de cambio nominal & TCN \\
\hline Índice de tipo de cambio real & ITCR \\
\hline Términos de intercambio & ТІ \\
\hline \multicolumn{2}{|l|}{ ESTADOS UNIDOS } \\
\hline Manufacturas & Maneu \\
\hline Volumen de la industria & Indeu \\
\hline Tasa de bonos del Tesoro a 3 meses nominal & ТВT3N \\
\hline Tasa de bonos del Tesoro a 3 meses real & твт3R \\
\hline \multicolumn{2}{|l|}{ ESTATAL } \\
\hline Manufacturas estatales & Manedo \\
\hline Índice de compras al menudeo & Indcme \\
\hline Índice de compras al mayoreo & Indcma \\
\hline Índice de ventas al mayoreo & Indvma \\
\hline Índice de ventas al menudeo & Indvme \\
\hline Producción de electricidad & Elec \\
\hline Asalariados sin prestaciones & Asalsinpres \\
\hline Tasa de desempleo de 12 a más años & De12mas \\
\hline Tasa de desempleo abierto estatal & Desedo \\
\hline Tasa de desempleo de 12 a 19 años & De12a19 \\
\hline Tasa de desempleo de 20 a 24 & De20a24 \\
\hline Tasa de desempleo de 25 a 34 & De25a34 \\
\hline Tasa de desempleo de 35 a 44 & De35a44 \\
\hline Tasa de desempleo de 45 a más & De45mas \\
\hline Tasa de desempleo de hombres & Desh \\
\hline Tasa de desempleo de mujeres & Desm \\
\hline Personal ocupado sin prestaciones & Ocsinprest \\
\hline
\end{tabular}


continuación...

\section{ESTATAL}

Población económicamente activa

Población económicamente activa hombres

PEA

Población económicamente activa mujeres

PEAH

Población no económicamente activa

Población no económicamente activa con experiencia

Población no económicamente activa sin experiencia

PEAM

Población no económicamente activa por quehaceres del hogar

NEA

Pneacex

Población no económicamente activa por disponibilidad

Pneasex

Pneacasa

Pneadisp

Pneanodis

Población no económicamente activa por no disponibilidad

Pneapens

Población no económicamente activa estudiantes

Trabajadores en negocios de 1 a 5 personas

Pneastu

Trest1a5

Trabajadores con menos de 1 salario mínimo trabajando más de 48 h a la semana

Trmensal48

ción para pronosticar su comportamiento futuro. Hasta donde sabemos, un análisis de esta naturaleza no se ha llevado a cabo para el Estado de México.

Entre nuestros hallazgos más importantes destacan los siguientes. En el ámbito nacional encontramos dos resultados importantes. Primero, aunque este aspecto no se evalúa formalmente, la comparación con los resultados de otros estudios sugiere la presencia de un cambio estructural en la dinámica cíclica de la economía mexicana a partir de la primera mitad de los años noventa: la naturaleza y magnitud de muchas relaciones cambia, por lo que es posible aventurar que la economía funciona ahora en mayor medida de acuerdo con los principios del mercado, sobre todo con respecto a las relaciones del ciclo con las variables monetarias y financieras y los precios. Esta afirmación se considera razonable dado que hacia la primera mitad de los años noventa pudieron haber madurado las transformaciones estructurales llevadas a cabo durante la década previa. Segundo, las variables monetarias nominales ofrecen evidencia de que el dinero puede ser exógeno, lo cual es consistente con la política monetaria con tipo de cambio flexible implementada en México a partir de 1995 y con los modelos convencionales de economía abierta. Un resultado que llama nuestra atención es la débil vinculación entre el indicador del ciclo nacional y la tasa de inflación, lo cual sugiere que la inflación puede ser dañina para el crecimiento solamente cuando se encuentra por encima de un cierto valor (estimado en alrededor de $8 \%$ por autores como Mejía, 2004, y Acevedo, 2006).

Para el ciclo mexiquense, encontramos dos resultados dignos de subrayar. Primero, se observa una gran similitud entre los ciclos de México y los del Estado de México, especialmente con respecto a su relación con las variables nacionales, sean domésticas o vinculadas al sector externo. La única diferencia impor- 
tante ocurre en lo referente a la sincronización del ciclo con la economía estadounidense: la economía mexicana está más fuertemente vinculada que la mexiquense. Dada la semejanza en la estructura productiva del país y del estado en la de división manufacturera, es posible que el distinto grado de sincronización se explique por diferencias visibles sólo a un nivel de desagregación mayor, de modo que las ramas en que se especializa el Estado de México estén menos vinculadas al exterior que las ramas nacionales correspondientes. De hecho, éste es un tema promisorio para la investigación futura. Segundo, resulta por demás interesante observar la baja correlación entre los indicadores locales y el ciclo estatal. Como se documenta en el texto, las relaciones de mayor magnitud se pueden catalogar como moderadas. Más aún, el perfil temporal sugiere que todas aquellas variables con las que hay una relación moderada siguen al indicador del ciclo. En suma, es posible afirmar que la dinámica cíclica de la economía mexiquense responde en buena medida a factores nacionales.

Finalmente, pensamos que a partir de este trabajo se desprenden distintas áreas de investigación. En primer lugar, destaca el uso de técnicas econométricas más elaboradas para estudiar con mayor detalle relaciones particulares entre el ciclo mexiquense y otras variables, como el empleo nacional y local, la captación bancaria y los precios locales, entre otras. En segundo lugar, se pueden analizar los efectos de las políticas nacionales sobre la dinámica productiva estatal. En ambos casos, la verificación formal de cambio estructural en las distintas relaciones documentadas en este texto es una de las tareas más importantes.

\section{Bibliografía}

Acevedo, Ernesto (2006), "Inflación y crecimiento económico en México: una relación no lineal”, Economía Mexicana, nueva época, xv(2), México, pp. 199-249.

Agénor, Pierre R., C. John McDermott y Eswar S. Prasad (2000), "Macroeconomic fluctuations in developing countries: some stylized facts", The World Bank Economic Review, XIV(2), Washington, pp. 251-285.

Ahumada, Hildegart y María L. Garegnani (2000), “Assesing HP filter performance for Argentina and U. S. macro aggregates”, Journal of Applied Economics, III(2), Buenos Aires, pp. 257-284. 
Alper, C. Emre (2002), "Business Cycles, Excess Volatility and Capital Floes: Evidence from México and Turkey", Russian and East European Finance and Trade, 38(4), Nueva York, pp. 22-54.

Altonji, Joseph y Mailer Ham (1990), "Variation in employment growth in Canada: The role of external, national, regional, and industrial factors", Journal of Labor Economics, 8, Chicago, pp. 198-236.

Braun, Miguel (2001), "Why is fiscal policy procyclical in developing countries?”, Universidad de Harvard, inédito.

Canova, Fabio (1998), "Detrending and business cycles facts", Journal of Monetary Economics, 41(3), Amsterdam, pp. 475-512.

Casar, José I., Gonzalo Rodríguez y Jaime Ros (1985), “Ahorro y balanza de pagos: un análisis de las restricciones al crecimiento económico de México", Economía Mexicana, 7, Centro de Investigación y Docencia Económicas, México, pp. 21-33.

Castañeda, Gonzalo (2000), "Internal capital markets and financial crisis. An explanation of the Mexican recovery after the 1995 crisis", Universidad de las Américas, Puebla, inédito.

Castillo, Ramón, Alejandro Díaz y Edna Fragoso (2004), “Sincronización entre las economías de México y Estados Unidos: el caso del sector manufacturero", Comercio Exterior, 54(7), México, pp.620-627.

Chadha, Bankim y Eswar Prasad (1994), "Are Prices Countercyclical? Evidence from the G7", Journal of Monetary Economics, Amsterdam, 34, pp. 239-257.

Clark, Todd (1998), "Employment fluctuations in U. S. regions and industries: The roles of national, region specific, and industry specific shocks", Journal of Labor Economics, 16, Chicago, pp. 202-219. 
Cottani, Joaquín A., Domingo F. Cavallo y M. Shabaz Khan (1990), "Real exchange rate behavior and economic performance in LDCs", Economic Development and Cultural Change, 39, Los Ángeles, pp. 61-76.

Cuevas, Alfredo, Miguel Messmacher y Alejandro Werner (2003), "Sincronización macroeconómica entre México y sus socios comerciales del TLCAN”, Banco de México, Documento de Investigación, núm. 2003-1.

Dornbusch, Rudiger y Alejandro Werner (1994), "Mexico: Stabilization, Reform and no Growth", Brookings Papers on Economic Activity, 1, Washington, pp. 253-315.

Erquizio, Alfredo (2007), "Ciclos económicos del Estado de México en el contexto regional: 1980-2005”, en P. Mejía Reyes, O. M. Rodríguez Pichardo y L. E. del Moral Barrera (coords.), Desempeño económico, producción manufacturera y políticas públicas en el Estado de México, Universidad Autónoma del Estado de México, Toluca, en prensa.

Gavin, Michael y Roberto Perotti (1997), "Fiscal policy in Latin America”, en Ben Bernanke y Julio Rotemberg (eds.), NBER Macroeconomics Annual 1997. MIT Press, Cambridge, Ma.

Herrera, Jorge (2004), "Business cycles in Mexico and the United States: do they share common movements?", Journal of Applied Economics, VII(2), Buenos Aires, pp. 303-323.

Hodrick, Robert J. y Edward C. Prescott (1997), "Postwar U. S. business cycles: an empirical investigation", Journal of Money, Credit and Banking, 29(1), Columbus, pp. 1-16.

Kamin, Steven B. y John H. Rogers (2000), "Output and real exchange rate in developing countries: an application to Mexico", Journal of Development Economics, 61, Yale, New Haven, pp.85-109.

Kose, M. Ayhan, Guy M. Meredith y Christopher M. Towe (2004), "How has NAFTA affected the Mexican economy? Review and evidence", Internacional Monetary Fund, Washington, Working Paper WP/04/59. 
Kydland, Finn E. y Edward C. Prescott (1990), "Business cycles: real facts and monetary myth", Federal Reserve Bank of Minneapolis Quarterly Review, XIV(2), Minnesota, pp. 3-18.

Loría, Eduardo y Gerardo Fuji (1997), “The balance of payment constraint to Mexico's economic growth 1950-1996", Canadian Journal of Development Studies, XVIII(1), Ottawa, pp. 119-137.

Lucas, Robert E. (1977), "Understanding business cycles”, Carnegie-Rochester Conference Series in Public Policy, 5, Pittsburgh, pp. 7-29.

Mejía-Reyes, Pablo (2003), "Regularidades empíricas en los ciclos económicos de México: producción, inversión, inflación y balanza comercial", Economía Mexicana, nueva época, XII(2), México, pp. 231-274.

Mejía-Reyes, Pablo (2004), "Modelling nonlinearities in the Mexican output growth", El Colegio Mexiquense, Documento de Investigación núm. 91, México.

Mejía-Reyes, Pablo, Elías E. Gutiérrez y Armando Pérez (2006), "Los claroscuros de la sincronización internacional de los ciclos económicos: evidencia sobre la manufactura de México", Ciencia Ergo Sum, 13(2), Universidad Autónoma del Estado de México, Toluca, pp. 133-142.

Navarrete-López, Emma Liliana (2001), Juventud y trabajo. Un reto para principios de siglo, México, El Colegio Mexiquense, Zinacantepec.

Negro, Marco del y Alejandro Ponce (1999), "Is North America an optimal currency area? Regional versus national shocks in the United States, Canada and Mexico", Instituto Tecnológico Autónomo de México, inédito.

Norrbin, Stefan y Don Schlagenhauf (1988), "An inquiry into the sources of macroeconomic fluctuations”, Journal of Monetary Economics, 22, Ámsterdam, pp. 43-70. 
Phillips, Keith R., Lucinda Vargas y Victor Zarnowitz (1996), "New tools for analysing the Mexican economy: indexes of coincident and leading economic indicators", Federal Reserve Bank of Dallas Economic Review, Economic Review, second quarter, Dallas, pp. 2-15.

Ponce, Alejandro (2001), "Determinantes de los ciclos económicos en México: ¿choques agregados o desagregados?", Gaceta de Economía, ITAM, México, año 6, 12, pp. 117155.

Ravn, Morten O. y Harald Uhling (1997), "On adjusting the HPfilter for the frequency of observations", London Business School, Londres, inédito.

Ron, Francisco E. (2001), "Ajuste dinámico y equilibrio entre la producción industrial y la actividad bursátil en México", Momento Económico, 118, Universidad Nacional Autónoma de México, México, pp. 21-38.

Sala-i-Martin, Xavier y Jeffrey Sachs (1992), "Fiscal federalism and optimum currency areas: Evidence from Europe and from United States", en Matthew B. Canzoneri, Paul R. Masson y Vittorio Grilli (eds.), Establishing a Central Bank: Issues in Europe and Lessons from the U.S., Cambridge University Press, Cambridge, pp. 195-219.

Samolyk, Katherine (1994), "Banking conditions and regional economic performance”, Journal of Monetary Economics, 34, Ámsterdam, pp. 259-278.

Stock, James H. y Mark W. Watson (1998), "Business cycle fluctuations in U. S. macroeconomic time series", National Bureau of Economic Research, Working Paper 6528, Washington.

Tornell, Aarón, Frank Westermann y Lorenza Martínez (2004), "NAFTA and Mexico's less-than-stellar performance", National Bureau of Economic Research, Working Paper 10289, Washington. 
Torres, Alberto (2002), "Estabilidad en variables nominales y el ciclo económico: el caso de México", La inflación en México. Gaceta de Economía, IтAм, México, tomo I, pp. 61-116.

Torres, Alberto y Óscar Vela (2003), "Trade integration and synchronization between business cycles of Mexico and the United States", North American Journal of Economics and Finance, 14(3), Ámsterdam, pp. 319-342.

Recibido: 14 de julio de 2006. Aceptado: 30 de noviembre de 2006.

Pablo Mejía Reyes. Profesor-investigador de la Facultad de Economía de la Universidad Autónoma del Estado de México. Es doctor en economía por la Universidad de Manchester, Reino Unido, y miembro del Sistema Nacional de Investigadores, Nivel I. Sus líneas de investigación son: fluctuaciones y crecimiento económicos, econometría aplicada y modelaje no lineal. Algunas de sus publicaciones son las siguientes: 1) "La sincronización de los ciclos económicos de México y Estados Unidos”, Investigación Económica, FE-UnAm, 258, 2006, pp. 15-45 (con Elías E. Gutiérrez Alva y Claudia A. Farías Silva). 2) "Los claroscuros de la sincronización internacional de los ciclos económicos: evidencia sobre la manufactura de México", Ciencia Ergo Sum, UAEM, 13(2), 2006, pp. 133-142 (con Elías E. Gutiérrez Alva y José A. Pérez Díaz). 3) "Comercio exterior y fluctuaciones cíclicas en la producción de cerveza en México”, Estudios Económicos Regionales y Sectoriales, EAAED, España, 5(2), 2005, pp. 81-110 (con Liliana Rendón Rojas). 4) "Ciclos económicos y sector externo en México. Evidencia de relaciones cambiantes en el tiempo", Estudios Económicos de Desarrollo Internacional, AEEADE, España, 5(1), 2005, pp. 65-92 (con E. E. Gutiérrez Alva y B. Cruz Flores).

Alberto Mejía Reyes. Asistente de Investigación de la Facultad de Economía de la Universidad Autónoma del Estado de México. Es egresado de la licenciatura en Relaciones Económicas Internacionales de la misma institución y ha sido asistente de investigación en El Colegio Mexiquense. Sus líneas de investigación son: fluctuaciones cíclicas y economía del Estado de México. 